\title{
Patterns of rabies cases in South Africa between 1993-2019, including the role of wildlife
}

\author{
Katja Koeppel ${ }^{1}$, Ockert van Schalkwyk ${ }^{2}$, and Peter Thompson ${ }^{3}$ \\ ${ }^{1}$ University of Pretoria Faculty of Veterinary Science \\ ${ }^{2}$ Government of South Africa \\ ${ }^{3}$ University of Pretoria
}

February 2, 2021

\begin{abstract}
Rabies is a global viral zoonosis endemic to South Africa, resulting in fatal encephalitis in warm blooded animals, including humans. The loss of human lives and economic losses in rural areas through loss of livestock are substantial. A review was conducted of all confirmed rabies cases in South Africa from 1993 to 2019, with a total of 11701 cases identified to species level to assess the wildlife plays in the epidemiology of rabies. A spatiotemporal cluster analysis using a discrete Poisson space-time probability model, accounting for underlying estimated dog and livestock densities, identified 13 significant clusters $(\mathrm{p}<0.05)$. These included four long-term clusters lasting more than 8 years in duration and seven short term clusters lasting less than 2 years, with the remaining two clusters being of intermediate length. Outside of these endemic clusters, wildlife outbreaks in the remainder of South Africa were often less than one and a half years in duration most likely due to the rapid decline of wildlife vectors, especially jackals associated with rabies infection. Domestic dogs accounted for $59.8 \%$ of cases, with domestic cats $(3.2 \%)$, livestock $(21.1 \%)$ and wildlife (15.8\%) making up the remainder of the cases. Yellow mongoose (Cynictis penicillate) was the most frequently affected wildlife species, followed by bat-eared fox (Otocyon megalotis), black-backed jackal (Canis mesomelas), meerkat (Suricata suricatta) and aardwolf (Proteles cristatus). Rabies in wildlife species followed different spatial distributions: black-backed jackal cases were more common in the north-western parts of South Africa, yellow mongoose cases more frequent in central South Africa, and bat-eared fox and aardwolf cases were more frequent in southern and western South Africa. Clusters often spanned several provinces, showing the importance of coordinated rabies control campaigns across administrative boundaries, and high-risk areas were highlighted for rabies in South Africa.
\end{abstract}

\section{Hosted file}

Rabies pattern SA2.pdf available at https://authorea.com/users/393256/articles/506911patterns-of-rabies-cases-in-south-africa-between-1993-2019-including-the-role-ofwildlife
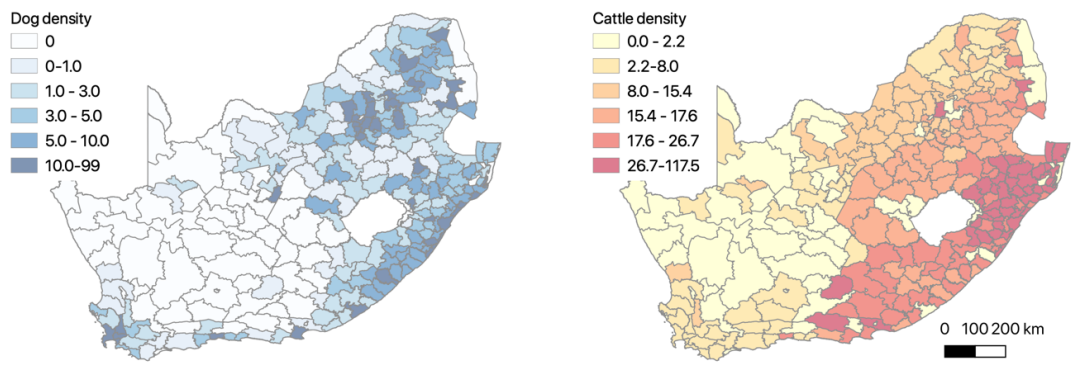


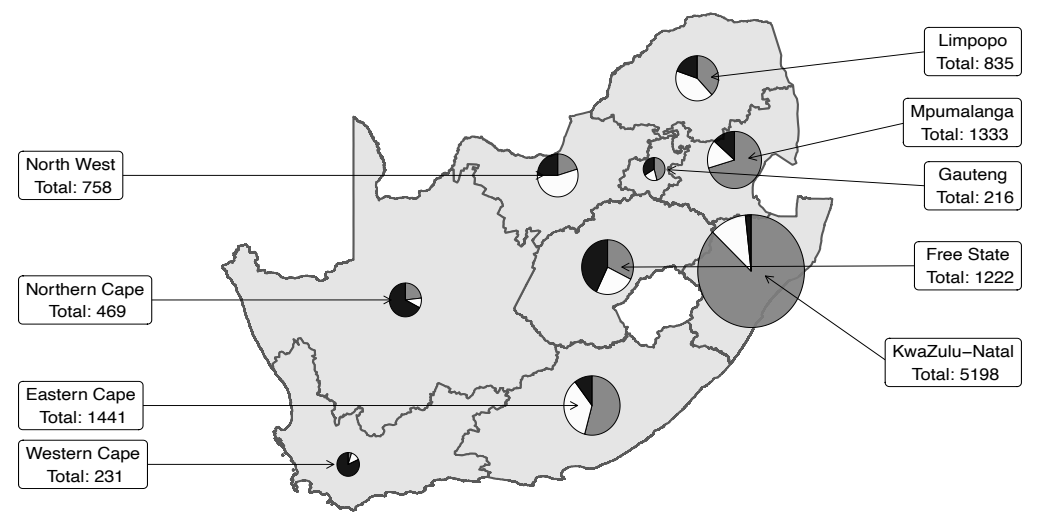

Companion $\square$ Livestock $\square$ Wildlife

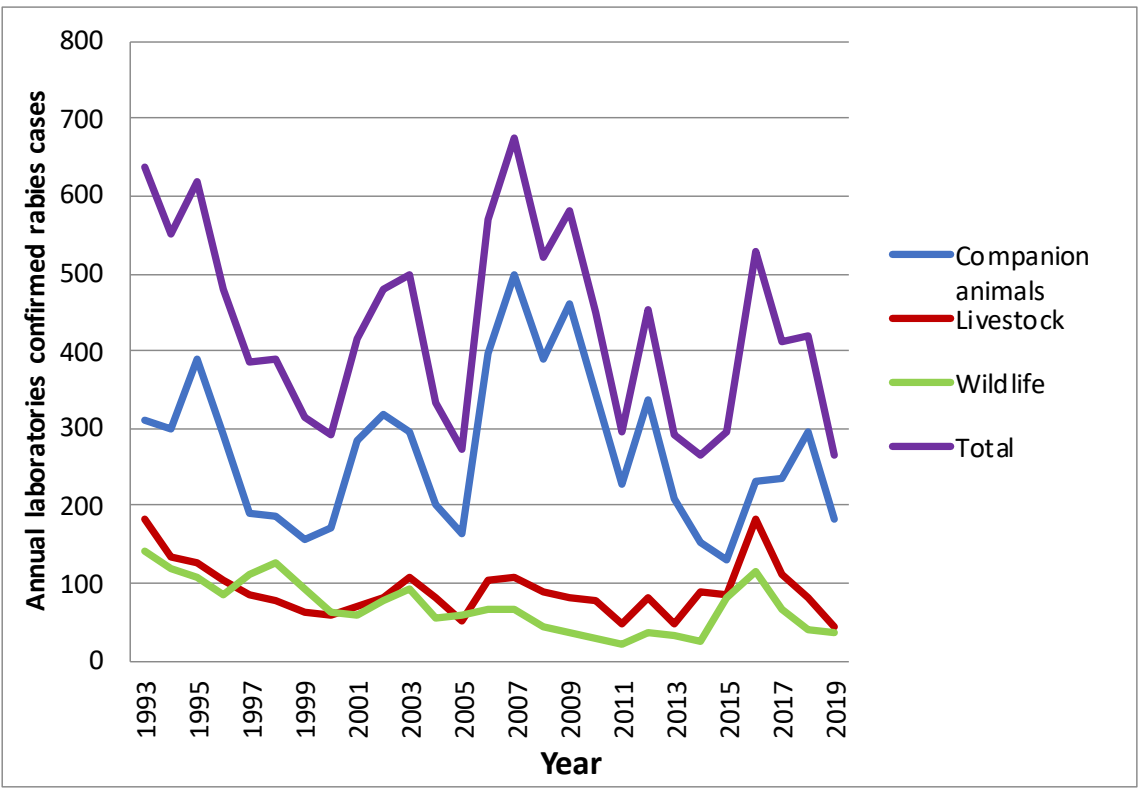



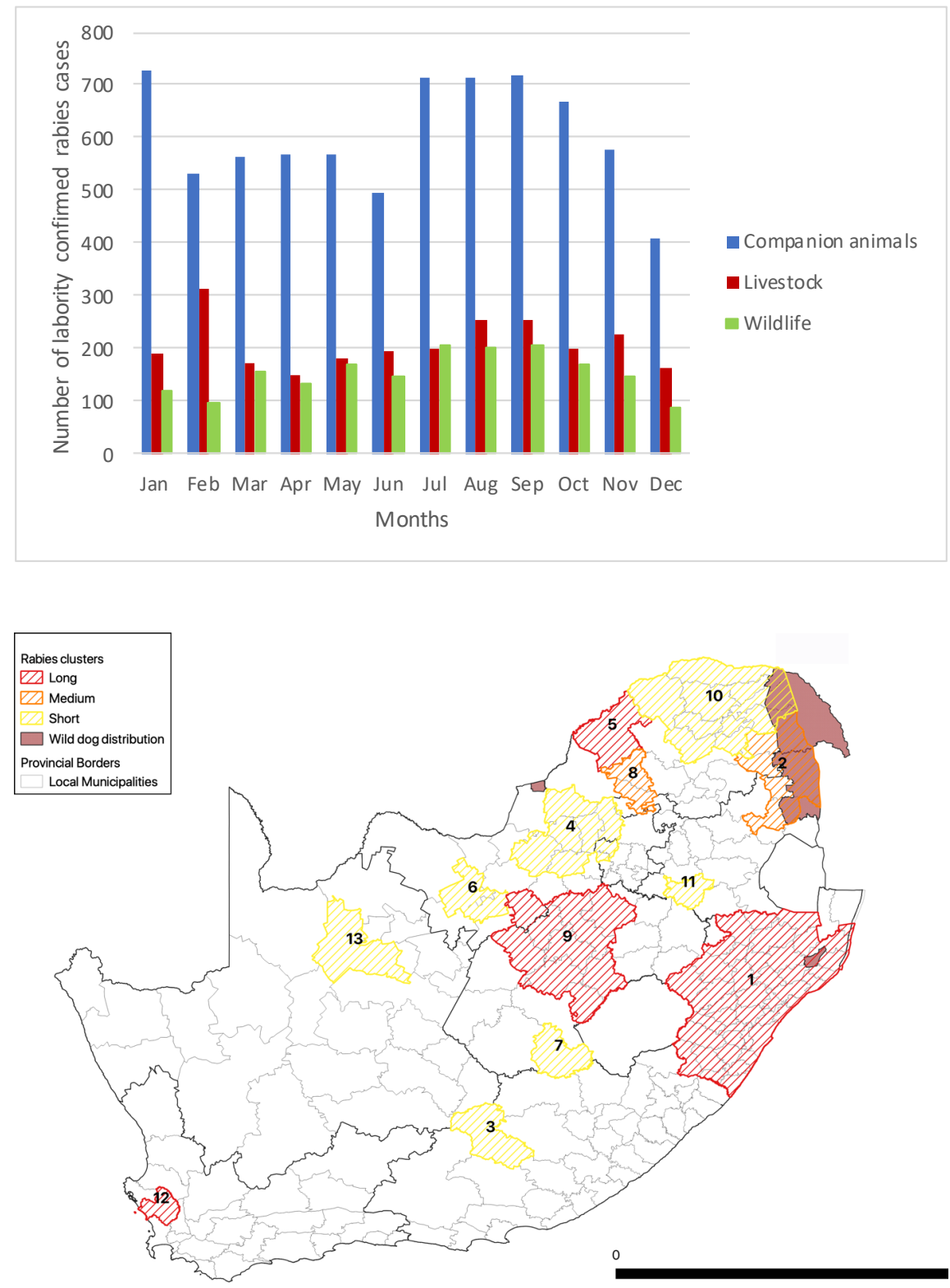

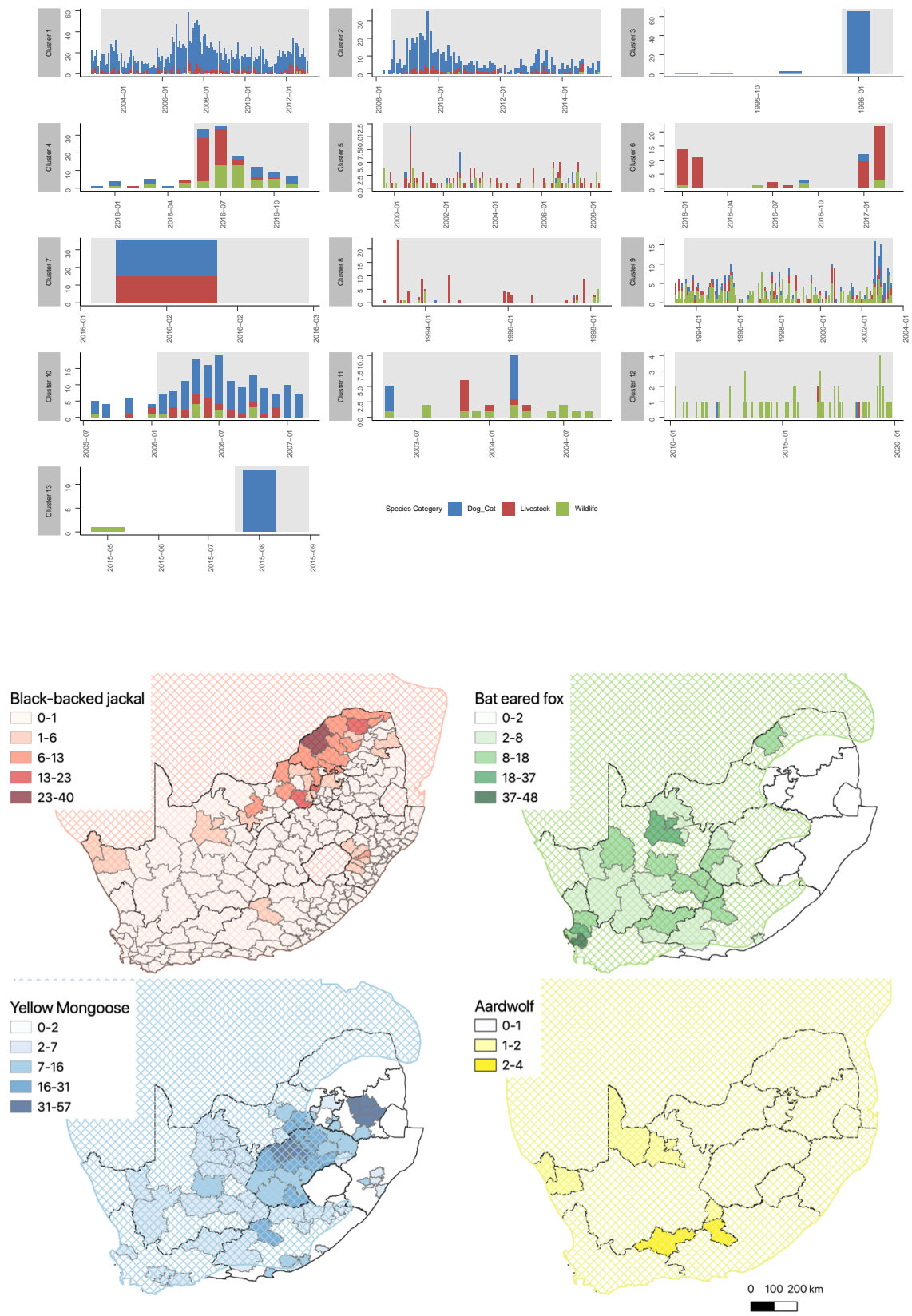

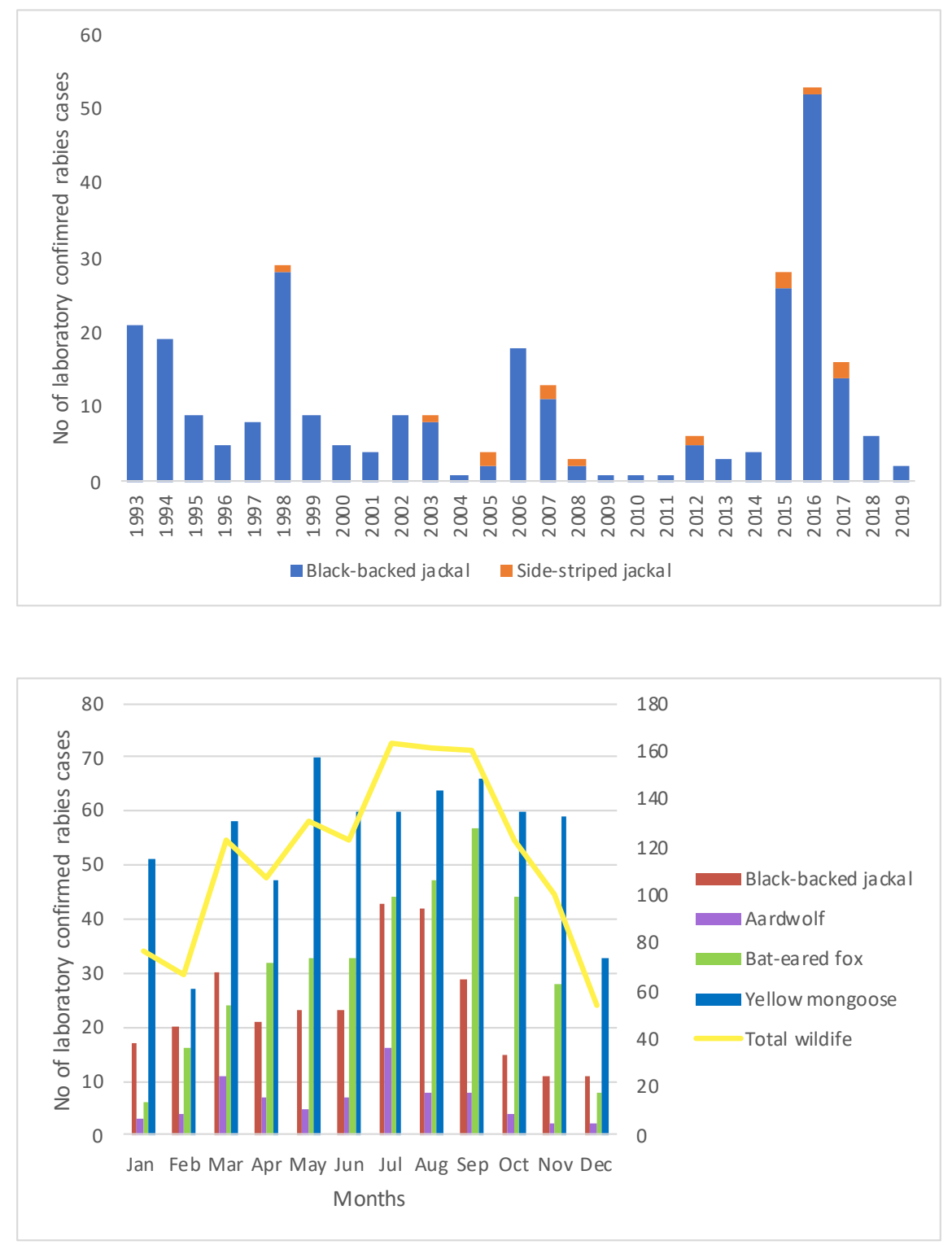


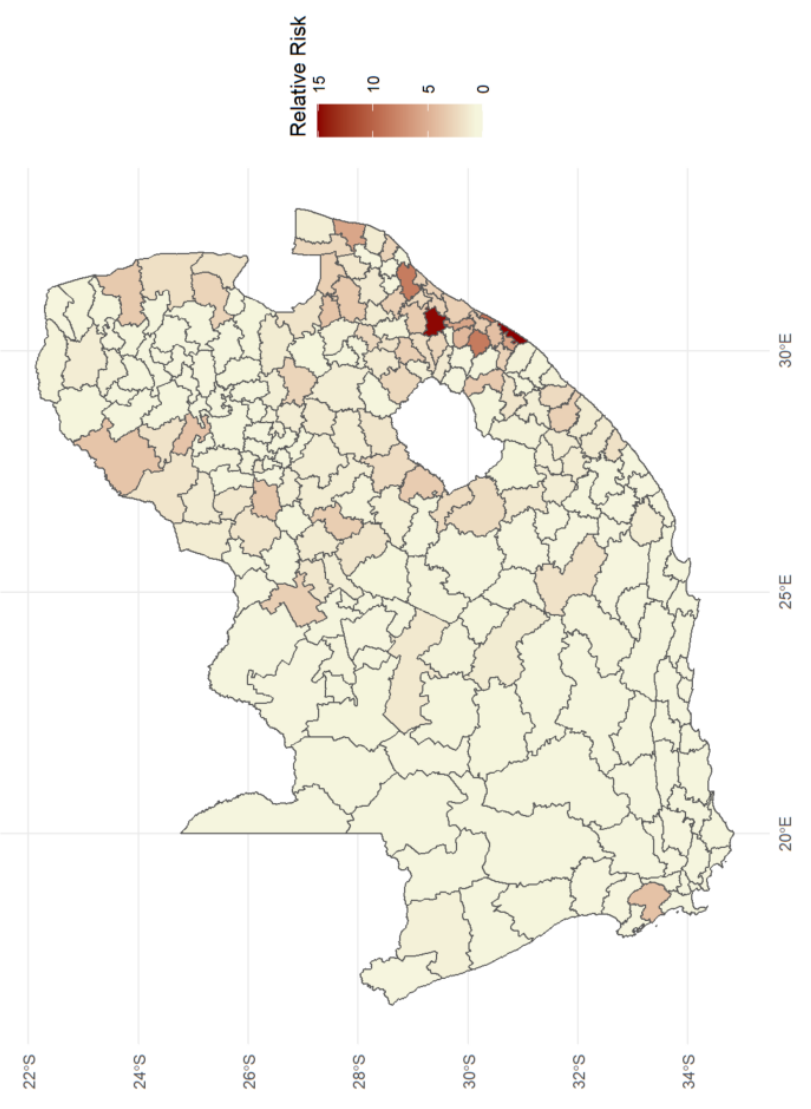

